\title{
Native mass spectrometry guided screening identifies hit fragments for HOP-HSP90 PPI inhibition
}

Clinton G. L. Veale, ${ }^{*[a]}$ Michaelone C. Vaaltyn, ${ }^{[b]}$ Maria Mateos-Jimenez, ${ }^{[c]}$ Ronel Müller, ${ }^{[a]}$ C. Logan Mackay, ${ }^{[c]}$ Adrienne L. Edkins ${ }^{[b]}$ and David J. Clarke ${ }^{[c]}$

[a] School of Chemistry and Physics, Pietermaritzburg Campus, University of KwaZulu-Natal, Private Bag X01, Scottsville, 3209, South Africa

[b] The Biomedical Biotechnology Research Unit (BioBRU), Department of Biochemistry and Microbiology Department, Rhodes University, Makhanda, South Africa

[c] EaStCHEM School of Chemistry, University of Edinburgh, Joseph Black Building, David Brewster Road, Edinburgh EH93FJ, UK

\begin{abstract}
Contemporary medicinal chemistry considers fragment-based drug discovery (FBDD) and inhibition of Protein-Protein Interactions (PPI), as important means of expanding druggable chemical space. However, the ability to robustly identify valid fragments and PPI inhibitors is an enormous challenge, requiring the application of sensitive biophysical methodology. Accordingly, in this study, we exploited the speed and sensitivity of nano-ESI native mass spectrometry to identify a small collection of fragments, which bind to the TPR2AB domain of HOP. Further biological assessment of a small selection of binding fragments showed that this binding translated into PPI inhibitory activity between the TPR2A domain of HOP and the HSP90$\boldsymbol{C}$ terminal domain. An in silico assessment of binding fragments, at the PPI interfacial region provided valuable structural insight for future fragment elaboration strategies, including the identification of losartan as a weak, albeit dose dependent inhibitor of the target PPI.
\end{abstract}

\section{Introduction}

Interactions between proteins are key regulators of cellular biology and provide opportunities to expand druggable chemical space. ${ }^{[1]}$ However, in contrast to classical enzyme and receptor targets, the interfaces between proteins are comparatively featureless, lacking the molecular topography present on the surfaces of proteins which bind small molecules. ${ }^{[2,3]}$ Additionally, protein interfaces are not associated with endogenous ligands ${ }^{[2]}$ which together creates a significant challenge for conventional approaches to drug discovery. While Protein-Protein Interactions (PPIs) have traditionally been considered difficult to drug, rapid growth in the application of biophysical methodology to inhibitor screening, has not only facilitated in the characterization of PPI interfaces, but has also made it possible to detect binding events between biomolecules and weakly interacting small molecules, thus ushering in the era of fragment based drug discovery (FBDD). ${ }^{[4,5]}$ The enhanced efficiency with which chemical space can be sampled in the absence of redundant chemical functionality is critical when considering the relatively featureless interface of a PPI.[ ${ }^{[6]}$ 
Whilst undeniably successful, the most commonly accessed biophysical methods for fragment screening, which include NMR, x-ray co-crystallography, ITC and SPR, have drawbacks associated with sample consumption, sensitivity and experimental set up. ${ }^{[7]}$ Whilst conventionally underutilised, the speed and sensitivity inherent to native mass spectrometry (MS) has seen it emerge as a powerful fragment screening technique, which is capable of detecting weak interactions between unlabelled and untethered small molecular fragments and target proteins in the gas phase..$^{[8-11]} \mathrm{MS}$ ligand binding experiments typically look to detect a molecular ion correlating to the apo protein in its native state (M) with the emergence of a new ion corresponding to the deconvoluted molecular mass of the proteinligand complex $(\mathrm{M} \cdot \mathrm{L})$ indicating ligand binding. Comparison of the relative intensities of these ion peaks provides a rough indication of binding strength. ${ }^{[12]}$ This comparatively simple and fast means of interpreting ligand binding makes native MS particularly suited to the screening portion of a FBDD campaign. While entropic contributions to binding free energy, such as hydrophobic interactions are weakened in the gas phase, enthalpic contributions to binding, which typically involve interactions between polar functional groups, survive the transmission into the gas phase. ${ }^{[13]}$ As such, native MS screening will tend to favour compounds with preferable solubility and polarity characteristics, thus improving the likelihood of retaining suitable physicochemical properties following entropy governed hit/lead optimization campaigns. ${ }^{[14]}$ However, despite this promise, to date, the application of MS based FBDD methodology to identify PPI inhibitors is limited. ${ }^{[15]}$

Our target in this study, the HSP70-HSP90 organising protein (HOP), is a co-chaperone which binds simultaneously to HSP70 and HSP90, acting as a scaffold for the transfer of partially folded client proteins between the two. Accordingly, HOP couples the de novo and stress-related protein folding pathways of HSP70, to the conformational regulation cycle of HSP90. ${ }^{[16]}$ Furthermore, formation of the HSP70-HOP-HSP90 ternary complex is required for assembly of the proteasome and therefore influences the efficiency of proteasomal-mediated protein turnover. ${ }^{[17]}$ While HOP is found in the uncomplexed form in normal cells, rapid production of primary metabolites associated with a cancerous environment, necessitates that HOP is found predominantly in high molecular weight complexes with HSP90 and HSP70. Therefore, inhibition of complex formation has been postulated as a selective anticancer target. ${ }^{[18,19]}$

The primary HOP-HSP90 binding interface is mediated by an interaction between the $C$-terminal MEEVD motif of HSP90, and the 'carboxylate clamp' region of the TPR2A domain of HOP. X-ray cocrystallisation of acetylated MEEVD (1) with TPR2A (PDB 1ELR, Figure 1A), revealed the network of salt bridges formed between Glu2 and Asp5 of MEEVD, with a series of basic amino acid residues located in the carboxylate clamp. Furthermore, Val4, was found to occupy a shallow hydrophobic pocket. ${ }^{[20]}$ Kawakami and co-workers exploited these structural features to develop a hybrid TPR peptide, designed to interact with the acidic MEEVD region of HSP90, and block its pro-oncogenic interaction with the TPR2A domain of HOP. ${ }^{[21]}$ This idea was expanded by McAlpine and co-workers, who reported a series of TPR inspired cyclic peptides, whose HSP90 interaction disrupted PPI formation, and HSP90's folding function. ${ }^{[22]}$ Pimienta et al. (2) followed more recently by Darby et al. (3) investigated the opposite face of the PPI, identifying small molecules which bound to TPR2A, and disrupted binding of the $C$-terminal MEEVD peptide, without explicitly demonstrating PPI inhibition. ${ }^{[23,24]}$ 
Given the potential of this target, we have also investigated inhibitors of this PPI, resulting in the identification of tetrazole peptide (4), whose binding to TPR2A resulted in disruption of the TPR2AHSP90 PPI in the sub-micromolar range. ${ }^{[25]}$

Given the efficiently of FBDD for probing chemical space, as well as the aforementioned advantages of native MS, we reasoned that a nano-ESI native MS fragment screening workflow might simultaneously demonstrate the utility of this technique for general PPI drug discovery, whilst providing a fruitful means of identifying HOP-HSP90 PPI fragment hits. Furthermore, the preferable physicochemical properties would facilitate elaboration into new classes of HOP-HSP90 PPI inhibitors, with potential for disrupting cellular proteostasis, and ultimately new cancer therapeutics.

\section{Results and Discussion}

With the aim of targeting the carboxylate clamp region of TPR2A, we assembled a 'cherry-picked' fragment library of 133 compounds, which all contained either a carboxylic acid or tetrazole functionality. Furthermore, as FBDD screening typically requires high fragment concentrations, we opted to conduct our screen against the combined TPR2AB domains. The TRP2B domain of HOP features its own carboxylate clamp, capable of binding EEVD motifs. However, structural features of the respective EEVD binding sites renders MEEVD five fold more selective for TPR2A over TPR2B. ${ }^{[26]}$ While the presence of an additional carboxylate clamp presented a potential challenge with respect to correlating the relative abundance of apoTPR2A and fragment bound species, the single fold of TPR2AB imparts greater stability to the protein, which we considered important in the presence of comparatively high concentrations of fragments. As a means of developing our native MS experimental conditions, we first looked to observe the gas phase association between the Ac-MEEVD-OH peptide and TPR2AB, where at a relative concentration of 1:1, we observed the apo TPR2AB (M) species, alongside the TPR2AB Ac-MEEVD-OH $(\mathrm{M} \cdot \mathrm{L})$ complex as a binding ratio of approximately 1: 0.6 (Figure 1B and C). In addition, we observed the formation of a minor $(\mathrm{M} \cdot 2 \mathrm{~L})$, corresponding to the binding of two Ac-MEEVD-OH peptides, possibly as a result of binding to TPR2B. Fragment screening was conducted at a final protein: fragment concentration of $6 \mu \mathrm{M}: 250 \mu \mathrm{M}$, where binding affinity was assessed only by the relative intensity of the $M \cdot L$ peak as compared to the apo $M$ peak (Figure 2 ). This assessment indicated that 29 fragments in the library bound to TPR2AB (Table 1 and 2). In total 104 fragments did not form an $(\mathrm{M} \bullet \mathrm{L})$ peak (S1 - S104, Table S1). Apart from providing insight into some structural requirements for binding, importantly, non-bonding fragments demonstrated that the MS methodology did not result in excessive surface (i.e. non-specific) adducting, which given the high fragment concentrations, and high surface contact area inherent to PPI interfaces was an initial concern. In most instances, fragment binding was accompanied by multiple binding events (e.g. $\mathrm{M} \cdot 2 \mathrm{~L}, \mathrm{M} \cdot 3 \mathrm{~L}$ ) in the mass spectra. However, given the previously mentioned observation, we were confident that this was not as a result of adducting, but rather as a result of interaction at both carboxylate clamps in addition to some nonspecific binding, which is common for high concentration fragment screening. ${ }^{[27]}$ An initial crude assessment of the screening results revealed that only fragments featuring an aromatic moiety bound to TPR2AB, with the majority of these possessing a six-membered aromatic ring attached either directly 
to the acidic functional group, or through a benzylic carbon. With a few exceptions, it was generally observed that fragments possessing a five membered aromatic ring attached directly to the acidic group; fragments possessing fused rings; and fragments featuring acidic side chains longer than two carbons tended not to bind (Table S1). Since MEEVD features two long chain glutamic acids residues, and no aromatics, these results were initially surprising. However, the hydrophobic Val4 binding site on TPR2A, is lined by two tyrosine residues (Tyr236 and Tyr248), while a third aromatic residue (Phe270) is located near the Glu2 binding region (Figure 1A). Accordingly, we postulate that whilst in solution, these aromatic residues interact with aromatic fragments, thus facilitating salt-bridge formation, which is maintained into the gas phase. Based on their rudimentary structural characteristics, the binding fragments were clustered into three groups, namely the benzoic acid analogues (F1 - F15 including pyridine containing analogues), phenyl acetic acid analogues (F16 - F24), and a third group (F25 F29) containing a mix of structural motifs, not fitting into groups 1 and 2. Group 1 contained four fragments in which a tetrazole ring acted as the acidic principle. Amongst these, F1 and F2 were decorated with dual or single halogen substituents ortho to the tetrazole, while in the case of fragments F5 and F6, the tetrazole ring was attached to a phenol or pyridyl ring respectively, with the additional heteroatoms positioned para to the tetrazole. Importantly, fragment F5 was particularly prone to multiple binding events. In agreement with these results, carboxylic acid containing fragments F7 - F9 contained both the ortho halogen and 4-hydroxyl motif, while fragments F10 - F12, contained a larger aromatic substituent at the ortho position, without a 4-hydroxyl. Interestingly, fragments S48 and S63, which are carboxylic acid containing analogues of $\mathbf{F 2}$, as well as a methoxy derivative (S68), did not bind, suggesting that the tetrazole is preferred for binding, and a para-hydroxyl or possibly a corresponding pyridine moiety enhances fragment binding. While further in the context of F10 - F12, the chemical properties of the ortho substituent plays an important role in fragment binding, including possibly their steric bulk and corresponding ability to disrupt the planarity between the phenyl ring and acid principle. Similarly, while both the picolinic (F3) and nicotinic (F13) acid analogues, which also featured either a halogen or phenyl substituents ortho to the acid moiety, were found to bind, the corresponding ortho methyl (S26 and S16) containing analogues, did not bind. While in general, an $M \cdot L$ peak was not detected for fragments featuring substituents meta to the acidic principle, two benzoic acid fragments (F14 and F15) were identified as TPR2AB binders. While fragment F14 was a comparatively weak binder, the piperidine moiety made it unique amongst the binding fragments.

As highlighted earlier, a para hydroxyl substituent was seemingly beneficial for fragment binding. While binding was also observed for methoxy analogue F4, the majority of fragments featuring larger substituents in this position (e.g. S4, S5, S52, S102), did not bind, indicating a possible size restriction at this position. The structure binding relationship (SBP) patterns observed amongst group 2 binders, largely mirrored those observed for group 1 , including a preference for halogens substituted ortho to the benzylic position (e.g. F16, F17, F18), or a para hydroxyl substituent (F19). Amongst this subset of fragments, a series of mandelic acid analogies (F20 - F24) emerged as prominent binders, with the alpha hydroxyl moiety presumably playing an important role. While comparisons of the binding data for F22 - F24, make it unclear whether ortho halogens enhanced binding, the binding ratio of F21 suggested that meta substation also disrupted binding to some extent. While no distinct patterns could 
be drawn from group 3, F26 - F28 all contained a gamma lactam, and were in general, closer representatives of a peptidomimetic small molecule. Most importantly F25 - F29 were structurally distinct from the fragments in groups 1 and 2 , and possibly occupied different space at the PPI interface, thus representing opportunity for fragments elaboration, or tethering.

Based on the MS binding assessment, a small subset of five promising fragments (F5, F16, F17, F22 and F24, Figure 3) were selected for in vitro analysis in an ELISA solid phase PPI assay between the C-terminal HSP90 domain and the TPR2A domain of HOP. The most effective PPI inhibitors (F5, F16 and F17), showed a dose dependant inhibition of the target PPI at concentrations typically utilised in early FBDD. Importantly, these data confirmed that the binding observed in the MS screen was, at least partially, as a result of TPR2A binding in a region required for PPI formation. While F24 showed some promising, albeit substantially reduced PPI inhibitory activity, its ortho difluoro analogue (F22) was inactive in this assay, suggesting that this fragment primarily binds in a region, which does not impact HSP90 PPI formation, including possibly the carboxylate clamp of TPR2B.

Having demonstrated promising PPI inhibition, we turned our attention to potential fragment elaboration strategies. To that end, we examined the predicted binding modes of our binding fragments in silico, using a previously reported method. ${ }^{[25]}$ This assessment indicated all group 1, 2 and some group 3 fragments preferentially orientated their phenyl rings inside the aromatic-hydrophobic VAL4 binding pocket, with the acidic moieties generally positioning themselves in close proximity to the, Gln298, Lys 301 and Arg305 cluster. From this orientation, the 4-hydroxyl moiety of the most active fragment F5 (Figure 4A), as well as F7 - F9 were predicted to form two additional $\mathrm{H}$-bond with Asn233 and Tyr248 located within the hydrophobic pocket. Ortho substituted fragments orientated their substituents in the direction of the ASP5 binding region, with the pyridine moiety of F10 (Figure 4B) predicted to form an electrostatic interaction with Lys229, which was unique amongst the docked fragments. In addition to pushing the tetrazole moiety closer toward the Gln298, Lys 301, Arg305 cluster, the benzylic carbon of F16 (Figure 4C) and F17, affords extra conformational flexibility, allowing the tetrazole to sample a greater region of this basic binding region. In contrast to F16 and F17, the carboxylic acid moieties of mandelic acid analogues (F20 - F24, Figure S1A) interacted only with Arg305. From a stereochemical point of view, the alpha hydroxyl moiety of the $R$ - and $S$ - isomers were predicted to interact with Arg305 and Asn264 respectively, with no obvious preference in the docking energies or scores. Like the group 1 and 2 fragments, the aromatic portion of isoindolinone containing F26, was predicted to bind in the VAL4 pocket, with the lactam carbonyl and acid moiety predicted to interact with Arg305 and GIn298 respectively (Figure S1B). While the aromatic portion of F27 and F28, were predicted bind in the vicinity of the VAL4 pocket, the acid moiety of both the $R$ - and $S$ - isomers of both fragments orientated in a similar position to the pyridine substituent of F10, forming a series of salt bridges with Lys229, Asn264 and Asn233 (Figure S1C). Finally, F29 had a unique predicted pose amongst the binding fragments, forming interactions with Arg305 and Asn308 in the GLU2 binding region. A structural overlay of the docked poses of group 1 and 2 fragments, with that of F29, (Figure 4D) resembled the ortho biphenyl tetrazole losartan (5). As such, we reasoned that 5 alongside valsartan (6) and irbesartan (7) might provide additional insight into future elaboration campaigns. While the predicted binding pose of all three compounds was not in close agreement with that of our fragments, all three compounds displayed 
a moderate and dose dependant disruption of the target PPI (Figure 5), and were important in conforming the promise of elaborated ortho substituted phenyl tetrazoles as HOP-HSP90 PPI inhibitors.

\section{Conclusion}

The ability to rapidly detect electrostatic interactions between protein targets and small molecules through native MS is becoming an increasingly useful tool in contemporary drug discovery. Furthermore, the inherent bias toward identifying compounds with preferable physicochemical properties offers additional value in this approach. Together, the sensitivity, speed and low sample consumption of native MS, particularly when coupled with nano-ESI in a multiwell format, makes a compelling case for greater application of native MS based screens for the identification of weakly binding fragments, as well as small molecules capable of binding to PPI interfaces. Despite this, there are only a limited number of reports pertaining to its application to FBDD and PPI drug discovery respectively. In this study, our nano-ESI, native MS screening strategy, identified a cohort of buffer soluble fragments, which bind to the TPR2AB domain of HOP. A small subset of these fragments were confirmed to disrupt the TPR2A - HSP90 C-terminal PPI, thus providing an important validation of this approach. In silico evaluation of the binding fragments at the TRP2A - MEEVD interface provided structural insight into their binding modes, which alongside the structural information of binding and non-binding fragments illuminated potential fragment elaboration strategies. Given the resemblance of the peptidomimetic losartan to a postulated elaborated fragment, we assessed three 'sartans' for their PPI inhibitory activity, where they were found to possess weak albeit dose dependant PPI inhibitory activity. While this activity can be considered low, previously reported small molecules, have generally only been shown to disrupt MEEVD binding, and not formal PPI disruption. Furthermore, and most importantly, this result confirmed the potential of ortho substituted phenyl tetrazoles, as scaffolds for inhibiting this PPI. As such, this will form the basis of a refined fragment elaboration strategy, which will combine promising structural features of binding fragments in order to further enhance PPI inhibitory activity.

\section{Experimental Section}

\section{Chemicals and Materials}

Small molecules used in this study were purchased from Key Organics, Sigma Aldrich and Alfa Aesar (purity $\geq 95 \%$ ). LCMS grade dimethyl sulfoxide (DMSO) and LCMS grade ammonium acetate $\left(\mathrm{NH}_{4} \mathrm{OAC}\right)$ were purchased from Sigma Aldrich. 


\section{Protein expression and Purification}

E. coli $\mathrm{BL21}$ (DE3) cells were transformed with the pGEX-3X-1400 plasmid containing the coding region for the TPR2AB region (residues 208-543) from murine HOP (which shares $98 \%$ amino acid sequence identity with human HOP). ${ }^{[28,29]}$ The production of GST-TPR2AB protein was induced by addition of $1 \mathrm{mM}$ isopropylthio- $\beta$-galactoside (IPTG) for 3 hours at $37^{\circ} \mathrm{C}$ and GST-TPR2AB purified by native GSHaffinity chromatography. ${ }^{[30]}$ The GST tag was removed to yield the untagged TPR2AB domain using the Factor Xa Cleavage Capture Kit (Merck Millipore) according to the manufacturer's instructions. The stages of protein purification and proteolytic cleavage of the GST tag was monitored by SDS-PAGE and Western blot analysis. ${ }^{[30]}$ The average yield of TPR2AB was $0.70 \pm 0.05 \mathrm{mg} / \mathrm{L}$.

\section{Sample Preparation}

Samples of TPR2AB were buffer exchanged into $100 \mathrm{mM} \mathrm{NH}_{4} \mathrm{OAc}$ using Zeba Spin Desalting Column (Thermo Fisher Scientific) prior to MS analysis. Concentration of TPR2AB screening solution was adjusted to $20 \mu \mathrm{M}$ with $100 \mathrm{mM} \mathrm{NH}_{4} \mathrm{OAc}$ buffer.

$50 \mathrm{mM}$ stock solutions of the fragments were prepared in LCMS grade DMSO. $2.5 \mathrm{mM}$ screening solutions ( $5 \%$ DMSO) were prepared by diluting $5 \mu$ aliquots of the DMSO stocks with $95 \mu \mathrm{l}$ of $100 \mathrm{mM}$ $\mathrm{NH}_{4} \mathrm{OAc}$ buffer.

For fragment binding analysis, $6 \mu \mathrm{l}$ and $2 \mu \mathrm{l}$ aliquots of the TPR2AB and fragment screening solutions respectively were mixed with a further $12 \mu \mathrm{l}$ of $100 \mathrm{mM} \mathrm{NH}_{4} \mathrm{OAc}$ buffer, to a final analysis concentration of $6 \mu \mathrm{M}$ TPR2AB, $250 \mu \mathrm{M}$ fragment, $0.5 \%$ DMSO. Samples were prepared in batches of 6 , and held at $4{ }^{\circ} \mathrm{C}$ prior to MS analysis.

\section{Native MS analysis}

Native MS and IM-MS data were obtained on both a Synapt-G2 Q-TOF (Waters). Ionisation was achieved using a NanoMate nESI infusion robot (TriVersa), sampling from a 96-well plate. All experiments were conducted under the same native MS conditions, i.e nanoelectrospay voltage of 1.54 $\mathrm{kV}$, cone voltage $100 \mathrm{~V}$, trap voltage of $5 \mathrm{~V}$ and a source temperature of $60^{\circ} \mathrm{C}$, while backing pressure was adjusted to 4.0 mbar. Final spectra were the sum of 240 scans collected over four minutes. MS data were processed using MassLynx v4.0 (Waters).

HSP90C-TPR2A PPI inhibition and in silico assessment were conducted using previously reported methods. ${ }^{[25]}$ 


\section{Acknowledgements}

The authors would like to acknowledge support from the Royal Society-Newton Fund (Grant No. NI160018), the BBSRC (Grant No. BB/R013993/1), Rhodes University, the University of KwaZulu-Natal flagship initiative, the National Research Foundation of South Africa (NRF, Grant Nos. 105829, 116305, 127224, 129262), the South African Research Chairs Initiative of the Department of Science and Innovation (DSI) and NRF (Grant No. 98566), and Future Leaders - African Independent Research (FLAIR), a partnership between the African Academy of Sciences and the Royal Society that is funded by the UK Government as part of the Global Challenge Research Fund (GCRF). RM and MCV gratefully acknowledge postdoctoral and postgraduate funding support from the NRF. We thank Dr Faye Cruickshank of the SIRCAMS mass spectrometry facility at the School of Chemistry, University of Edinburgh for technical support, Ms Kim Germiquet for sartan samples and the Centre for High Performance Computing (CHPC) for access to Schrodinger's modelling suite.

Keywords: Fragment Based Drug Discovery $•$ Native Mass Spectrometry $•$ PPI Inhibitors $\cdot$ Heat Shock Protein $90 \cdot$ HSP70-HSP90 Organising Protein

[1] B. O. Villoutreix, C. M. Labbe, D. Lagorce, G. Laconde, O. Sperandio, Curr. Pharm. Des. 2012, $18,4648-4667$.

[2] J. A. Wells, C. L. McClendon, Nature 2007, 450, 1001-1009.

[3] S. Surade, T. L. Blundell, Chem. Biol. 2012, 19, 42-50.

[4] C. Jacquemard, E. Kellenberger, Expert Opin. Drug Discov. 2019, 14, 413-416.

[5] M. Zhou, Q. Li, R. Wang, ChemMedChem 2016, 11, 738-756.

[6] A. P. Turnbull, S. M. Boyd, B. Walse, Res. reports Biochem. 2014, 4, 13-26.

[7] D. Shiu-Hin Chan, A. J. Whitehouse, A. G. Coyne, C. Abell, Essays Biochem. 2017, 61, 465473.

[8] H. Vu, L. Pedro, T. Mak, B. McCormick, J. Rowley, M. Liu, A. Di Capua, B. Williams-Noonan, N. B. Pham, R. Pouwer, et al., ACS Infect. Dis. 2018, 4, 431-444.

[9] P. K. Chrysanthopoulos, P. Mujumdar, L. A. Woods, O. Dolezal, B. Ren, T. S. Peat, S. A. Poulsen, J. Med. Chem. 2017, 60, 7333-7349.

[10] C. G. L. Veale, M. Mateos Jimenez, C. L. L. Mackay, D. J. D. J. Clarke, Rapid Commun. Mass Spectrom. 2020, 34, e8570.

[11] H. Vu, C. Roullier, M. Campitelli, K. R. Trenholme, D. L. Gardiner, K. T. Andrews, T. SkinnerAdams, G. J. Crowther, W. C. Van Voorhis, R. J. Quinn, ACS Chem. Biol. 2013, 8, 26542659.

[12] L. Pedro, R. Quinn, Molecules 2016, 21, 984.

[13] L. A. Woods, O. Dolezal, B. Ren, J. H. Ryan, T. S. Peat, S.-A. Poulsen, J. Med. Chem. 2016, 59, 2192-2204.

[14] G. Klebe, Nat. Rev. Drug Discov. 2015, 14, 95-110.

[15] A. F. M. Gavriilidou, F. P. Holding, J. E. Coyle, R. Zenobi, SLAS Discov. 2018, 23, 951-959.

[16] S. Alvira, J. Cuéllar, A. Röhl, S. Yamamoto, H. Itoh, C. Alfonso, G. Rivas, J. Buchner, J. M. 
Valpuesta, Nat. Commun. 2014, 5, 5484.

[17] K. Bhattacharya, L. Weidenauer, T. M. Luengo, E. C. Pieters, P. C. Echeverría, L. Bernasconi, D. Wider, Y. Sadian, M. B. Koopman, M. Villemin, et al., Nat. Commun. 2020, 11, 5975.

[18] H. Kubota, S. Yamamoto, E. Itoh, Y. Abe, A. Nakamura, Y. Izumi, H. Okada, M. lida, H. Nanjo, H. Itoh, et al., Cell Stress Chaperones 2010, 15, 1003-1011.

[19] A. L. Edkins, Top. Med. Chem. 2016, 19, 21-54.

[20] C. Scheufler, A. Brinker, G. Bourenkov, S. Pegoraro, L. Moroder, H. Bartunik, F. U. Hartl, I. Moarefi, Cell 2000, 101, 199-210.

[21] T. Horibe, M. Kohno, M. Haramoto, K. Ohara, K. Kawakami, J. Transl. Med. 2011, 9, 8.

[22] M. N. Rahimi, S. R. McAlpine, Chem. Commun. 2019, 55, 846-849.

[23] G. Pimienta, K. M. Herbert, L. Regan, Mol. Pharm. 2011, 8, 2252-2261.

[24] J. F. Darby, L. R. Vidler, P. J. Simpson, B. Al-Lazikani, S. J. Matthews, S. Y. Sharp, L. H. Pearl, S. Hoelder, P. Workman, Sci. Rep. 2020, 10, 16000.

[25] C. G. L. Veale, M. Mateos-Jiménez, M. C. Vaaltyn, R. Müller, M. P. Makhubu, M. Alhassan, B. G. de la Torre, F. Albericio, C. L. Mackay, A. L. Edkins, et al., Chem. Commun. 2021, 57, 10919-10922.

[26] A. B. Schmid, S. Lagleder, M. A. Gräwert, A. Röhl, F. Hagn, S. K. Wandinger, M. B. Cox, O. Demmer, K. Richter, M. Groll, et al., EMBO J. 2012, 31, 1506-1517.

[27] P. Kirsch, A. M. Hartman, A. K. H. Hirsch, M. Empting, Molecules 2019, 24, 4309.

[28] J. Van Der Spuy, B. D. Kana, H. W. Dirr, G. L. Blatch, Biochem. J. 2000, 345, 645-651.

[29] S. Makumire, T. Zininga, J. Vahokoski, I. Kursula, A. Shonhai, PLoS One 2020, 15, e0226657.

[30] S. J. Beckley, M. C. Hunter, S. N. Kituyi, I. Wingate, A. Chakraborty, K. Schwarz, M. P. Makhubu, R. P. Rousseau, D. K. Ruck, J. A. de la Mare, et al., Int. J. Mol. Sci. 2020, 21, 3152. 
Figures and Tables<smiles></smiles>
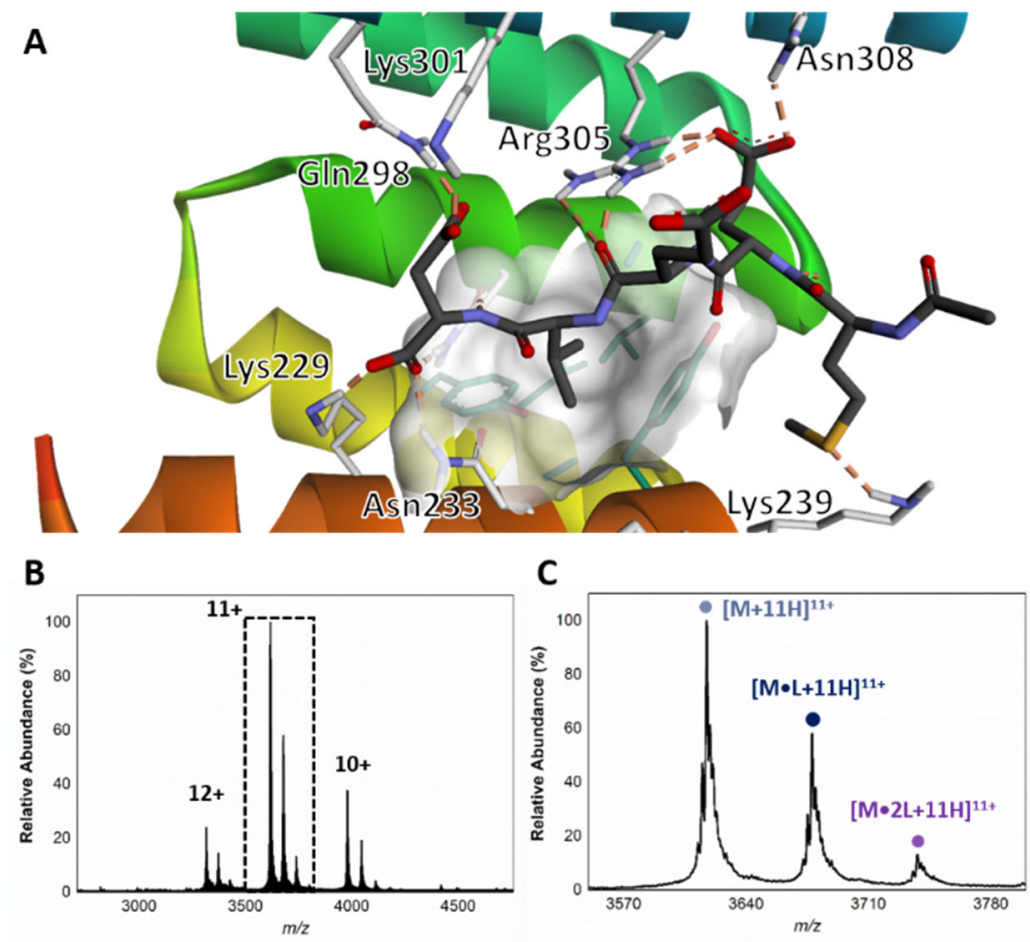

Figure 1. A. X-ray co-crystal of Ac-MEEVD-OH bound to the TPR2A domain of HOP (PDB 1ELR). Glu2 and Asp5 form a series of salt bridges with Lys229, Asn233, Gln298, Lys301, Arg305 and Asn308 residues, while Val4 occupies a hydrophobic pocket (white surface) 

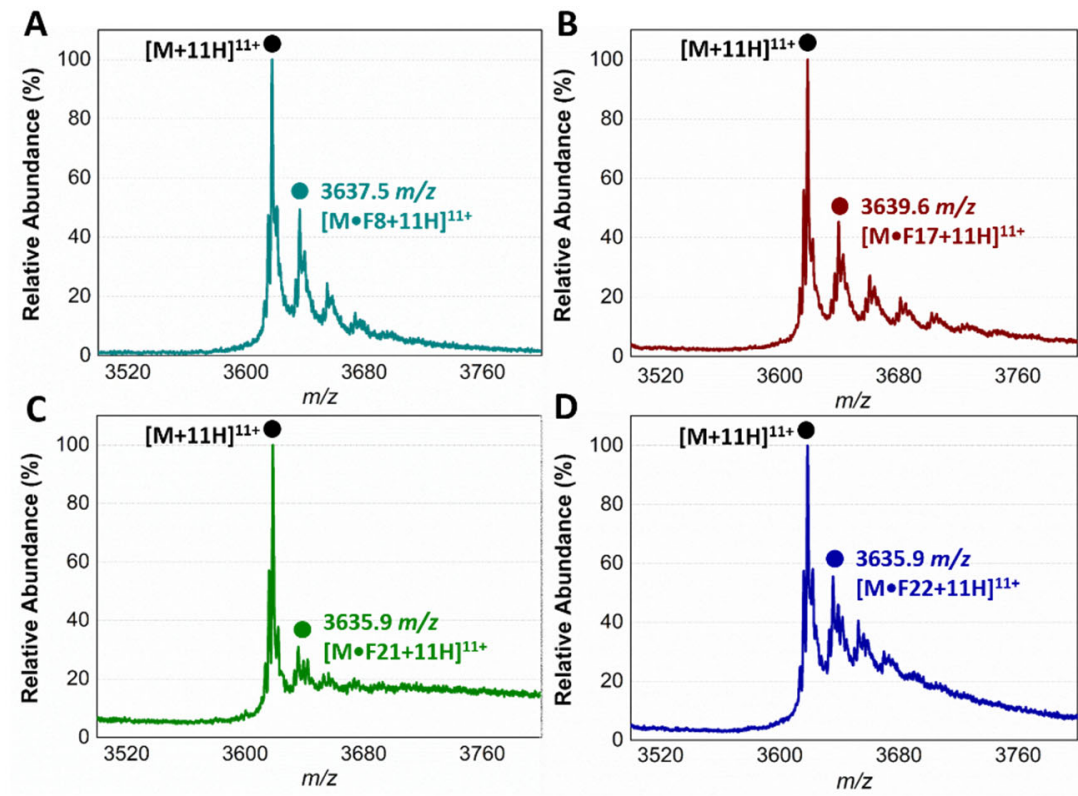

Figure 2. Expanded region of the $11^{+}$charge state, of a buffered mixture of TPR2AB with four selected binding fragments, F8 (A), F17 (B), F21 (C) and F 22 (D). Binding ratio was estimated based on the relative abundance of apo TPR2AB and TPR2A - fragment complex and shown in Tables 1 and $\mathbf{2}$

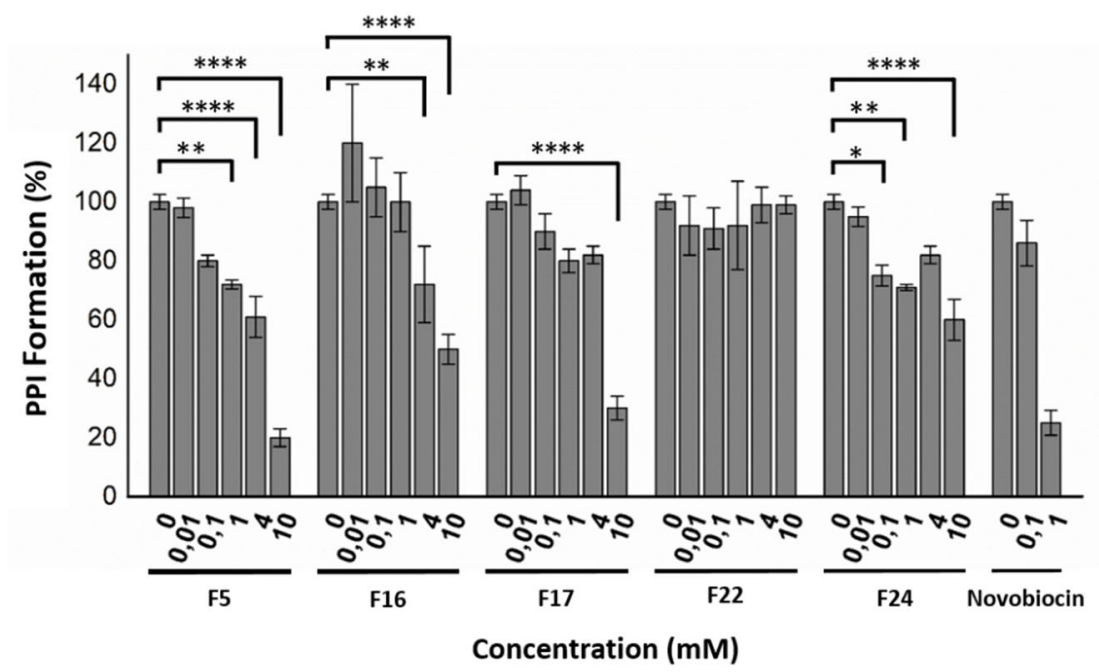

Figure 3. Solid phase PPI ELISA assay, between the HOP TRP2A domain and the HSP9O Cterminal domain, in the presence of several selected fragments. F5, F16 and F17 in particular displayed the most promising dose dependant PPI inhibition. ${ }^{*} p<.05 ;{ }^{* *} p<.01$ 

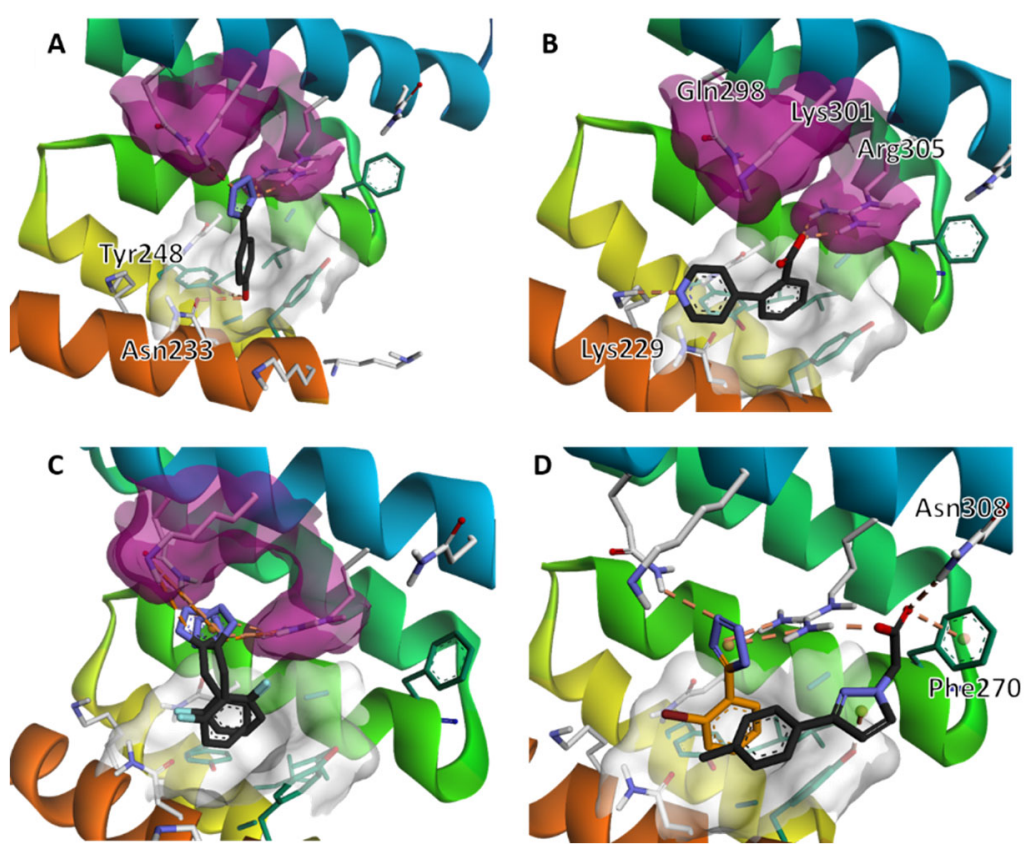

Figure 4 A - C. Docked binding pose of F5, F10 and F16 respectively. Group 1 and 2 fragments were predicted to bind at the hydrophobic Val4 binding pocket (white surface), with their acidic moieties predicted to interact with the basic, Gln298, Lys 301 and Arg305 cluster purple). D Overlay of the binding poses of F2 and F29, whose combined structure resembles losartan (5)

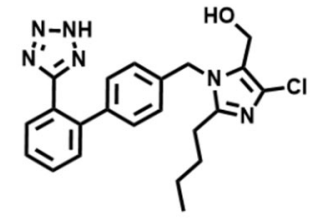

5. Losartan<smiles>CCCC(=O)N(Cc1ccc(-c2ccccc2-c2nn[nH]n2)cc1)C(C(=O)O)C(C)C</smiles>

6. Valsartan

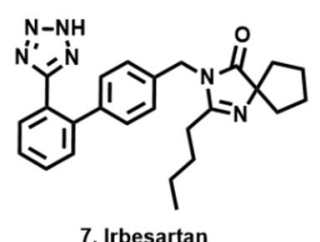

7. Irbesartan

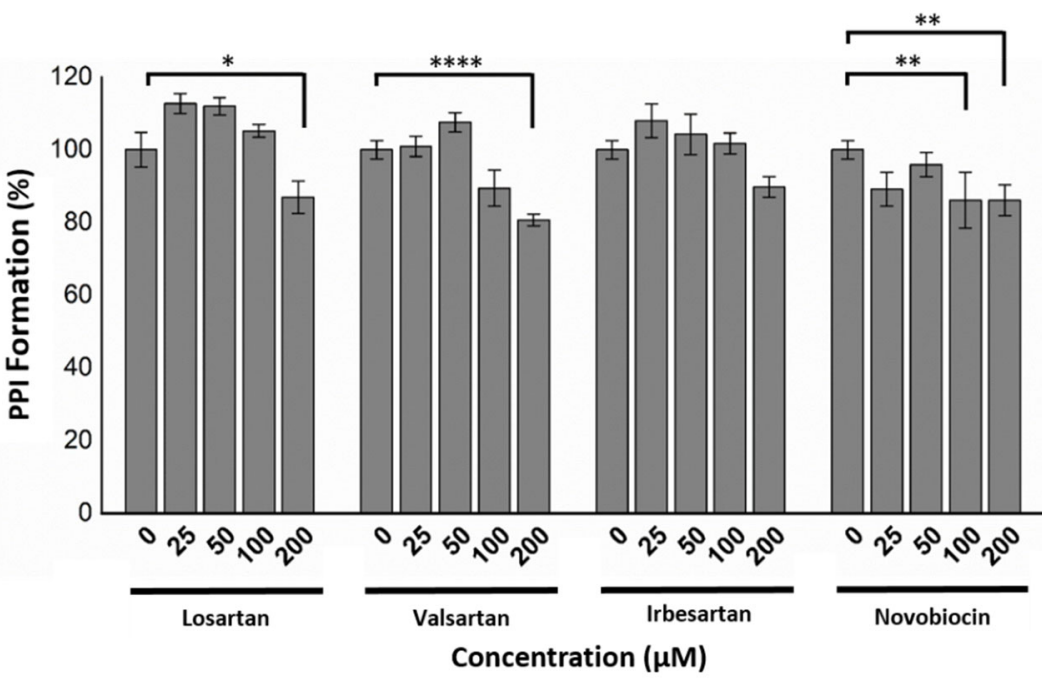

Figure 5. Solid phase PPI ELISA assay, between HOP TRP2A domain and the HSP9O Cterminal domain. Both losartan and valsartan displayed statistically significant PPI inhibitory activity at $200 \mu \mathrm{M} .{ }^{*} \mathrm{p}<.05 ;{ }^{* *} \mathrm{p}<.01 ; * * \mathrm{p}<.001 ; * * * * \mathrm{p}<.0001$. 
Table 1. Structures and Binding Ratios of Group 1 Binding Fragments

(1) $1: 0.50$ (1: $1: 0.50$


Table 2. Structures and Binding Ratios of Group 2 and 3 Binding Fragments

(16)




\section{Supplementary Information}

Mass Spectra of binding fragments
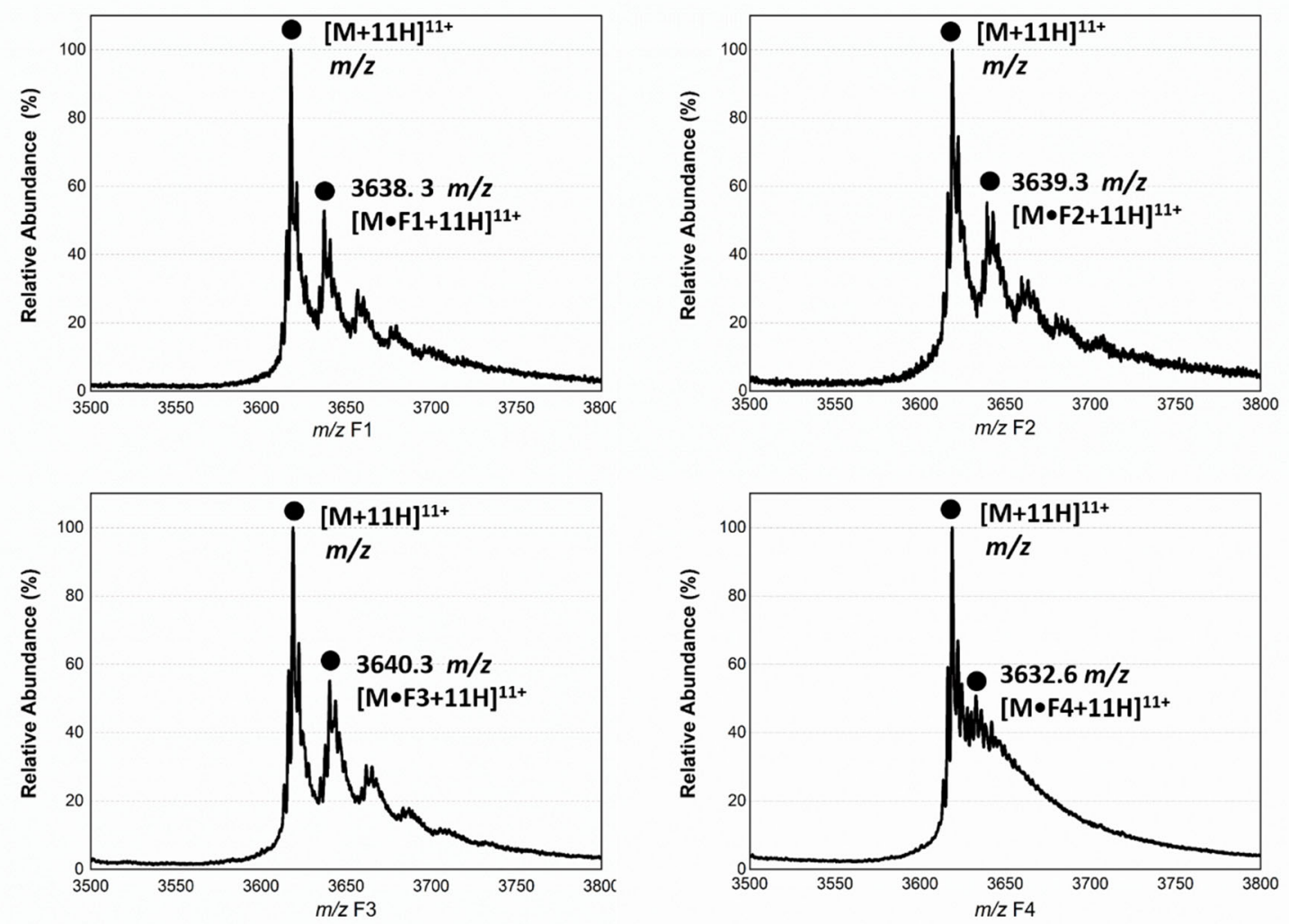

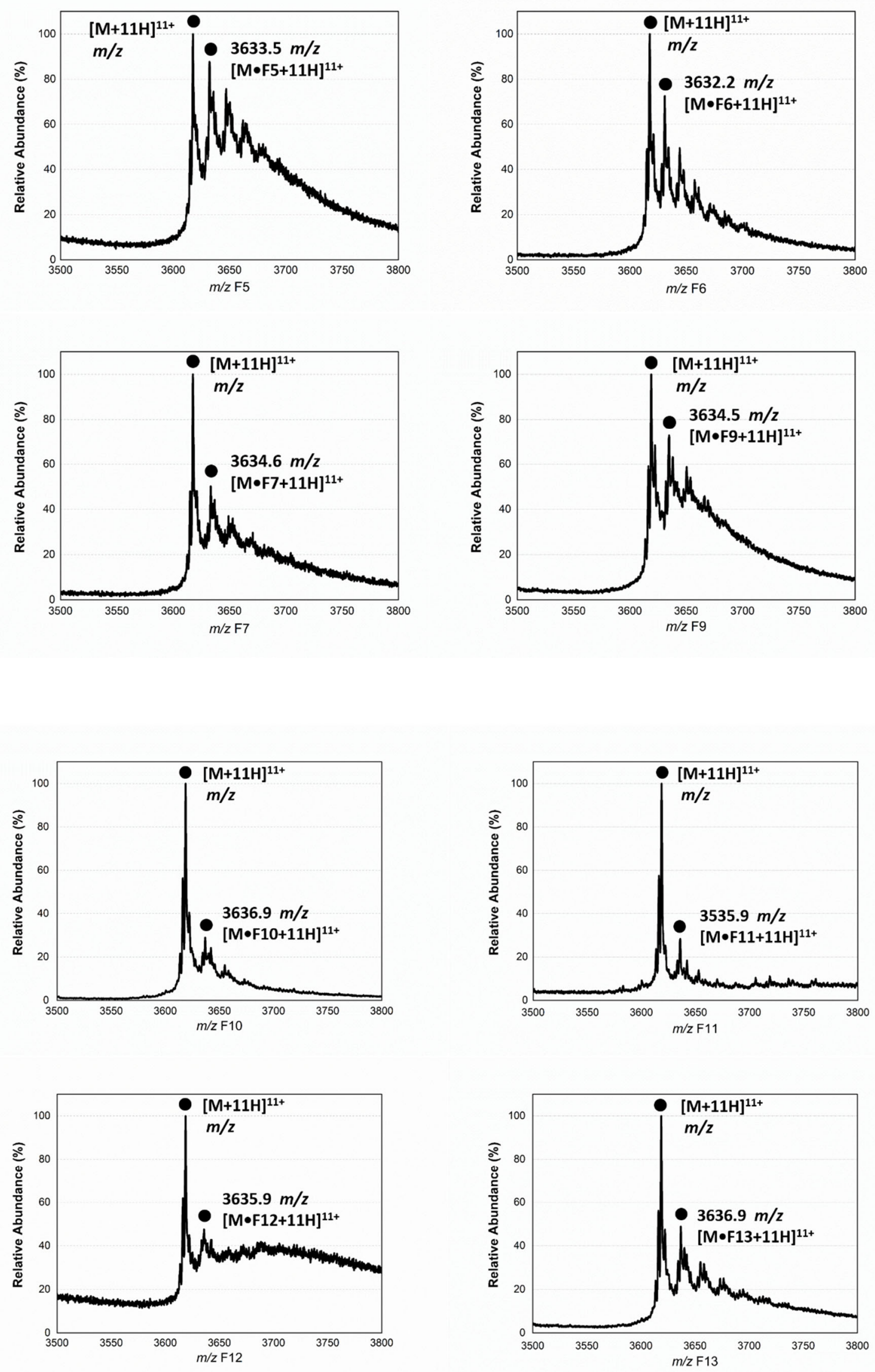

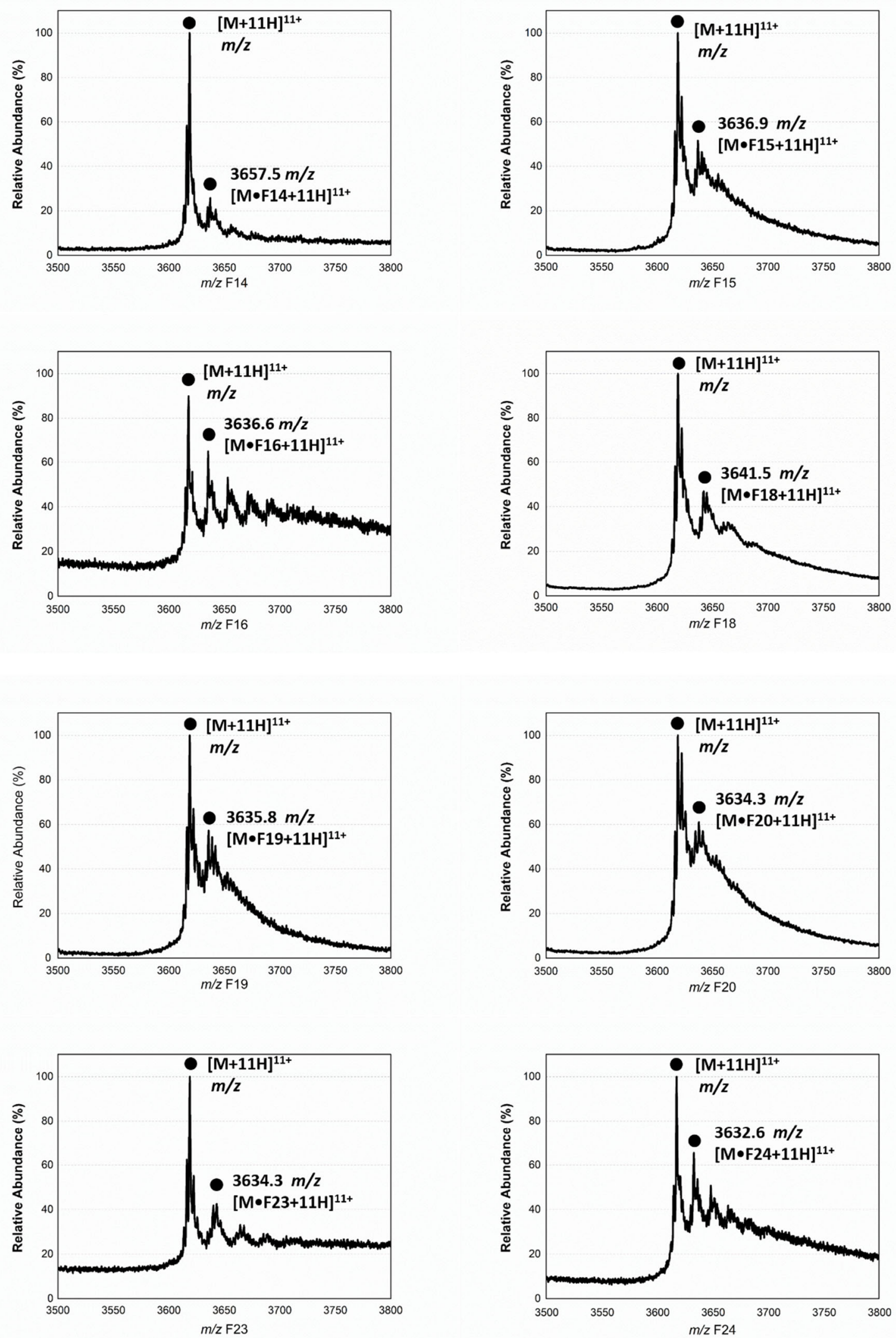

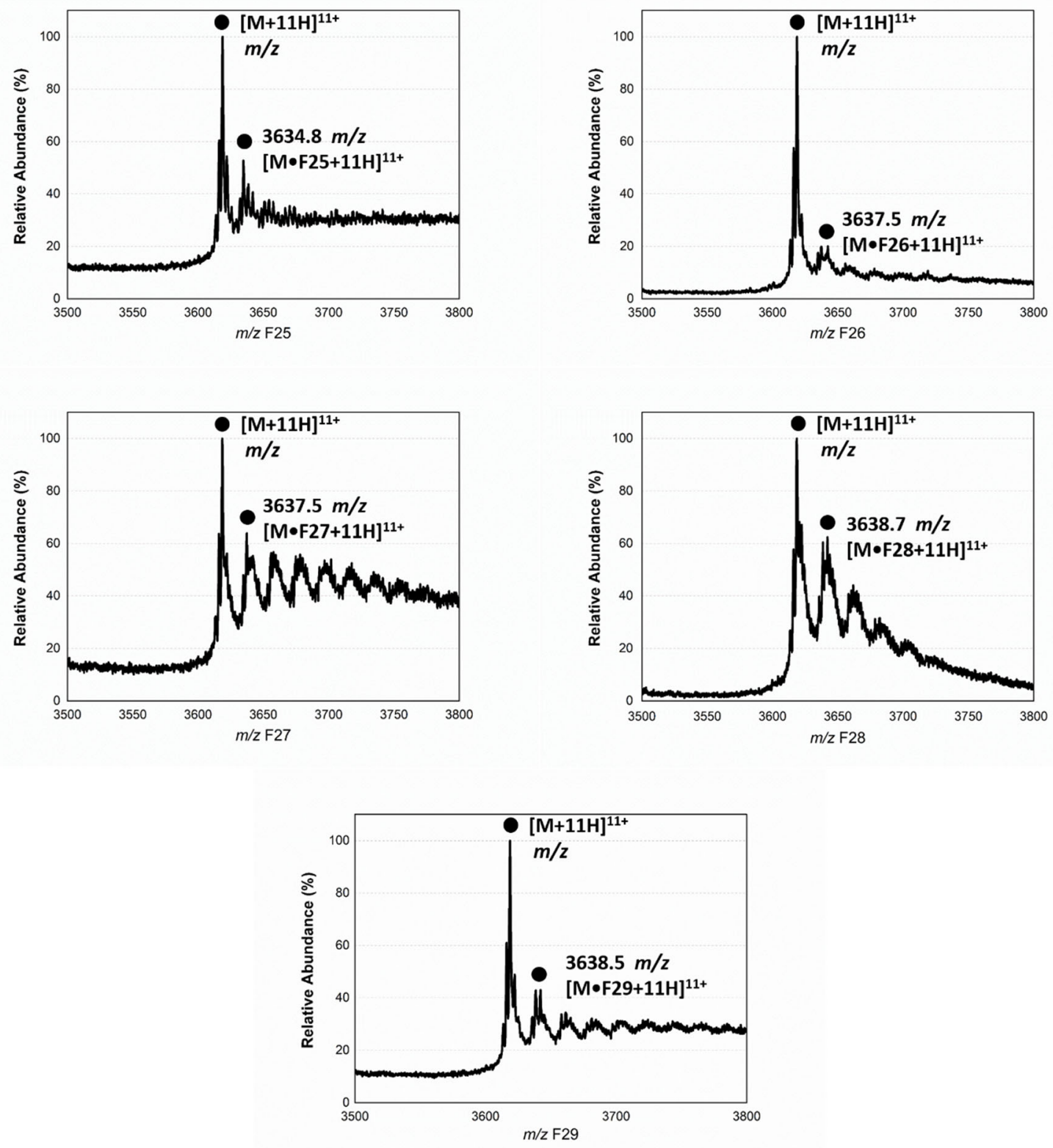


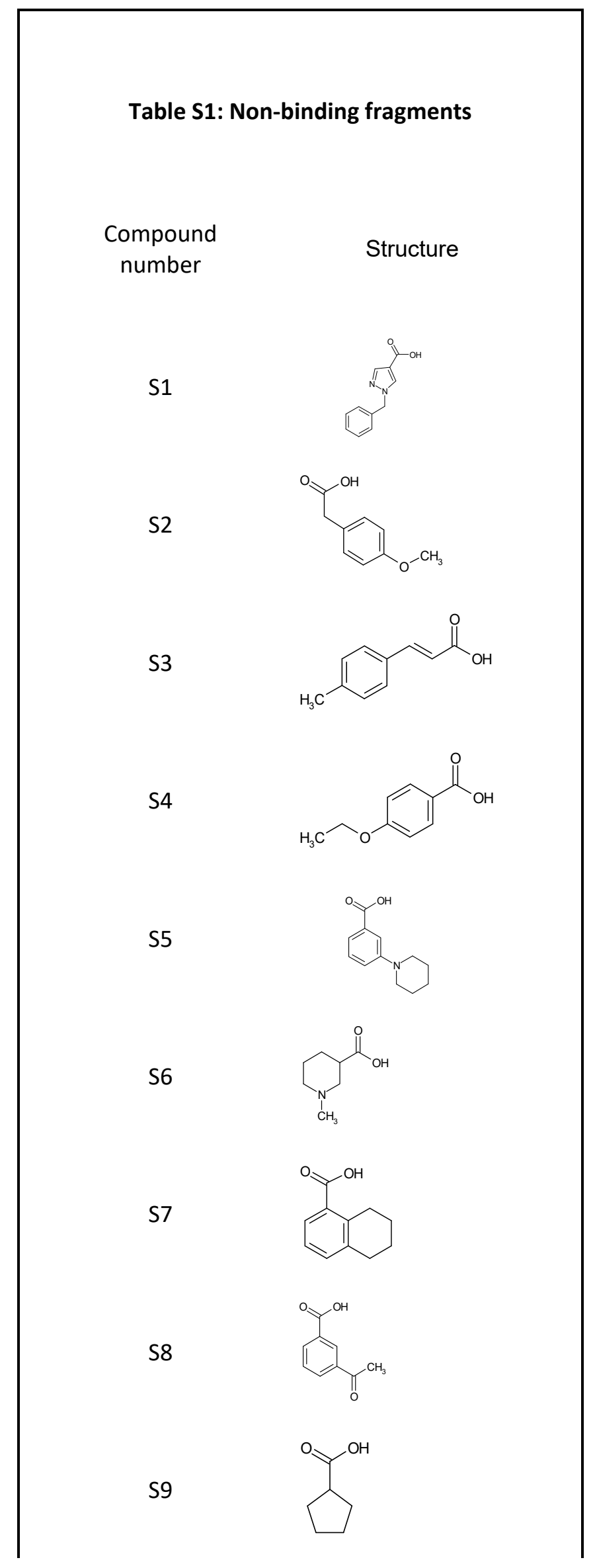


S10

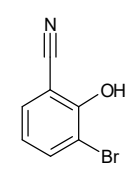

S11

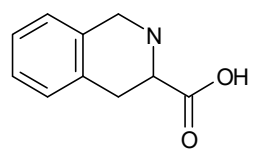

S12<smiles>CC(C)C1CCC(C(C)C)C1c1ccccc1</smiles>

S13<smiles>O=C(O)C1CCCc2ccccc21</smiles>

S14<smiles>O=C(O)Cc1cccc(O)c1</smiles>

S15<smiles>CCc1ccc(CC(C)C)cc1</smiles>

S16<smiles>Cc1ncccc1C(=O)O</smiles>

S17<smiles>O=C(O)CCn1ccnc1</smiles>

S18

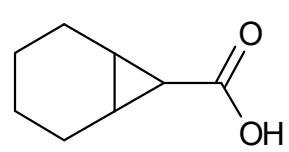

S19<smiles>Cn1cc(Cl)c(C(=O)O)n1</smiles>

S20<smiles>Cc1cc(C(=O)O)nn1C</smiles> 
S21<smiles>O=C(O)c1ccoc1</smiles>

S22<smiles>CC(C)c1ccc(CC(=O)O)cc1</smiles>

S23<smiles>O=C(O)C1(c2ccccc2)CCCC1</smiles>

S24

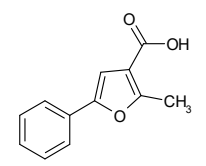

S25<smiles>Cn1cncc1C(=O)O</smiles>

S26

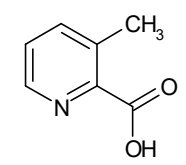

S27<smiles>O=C(O)c1cccc(-n2cccc2)c1</smiles>

S28<smiles>O=C(O)c1ccnn1-c1ccccc1</smiles>

S29<smiles>O=C(O)CCC12CC3CC(CC(C3)C1)C2</smiles>

S30

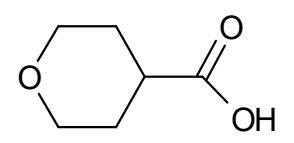

S31<smiles>O=C(O)C1CC=CC1</smiles> 
S32

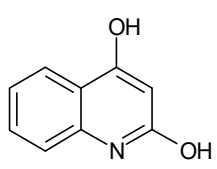

S33

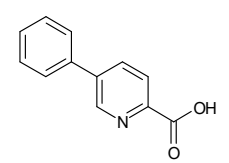

S34

(1)

S35

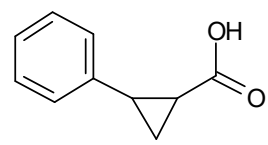<smiles>O=C(O)c1ccnc(Cl)c1</smiles>

S37

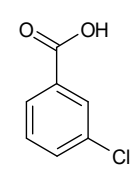

S38

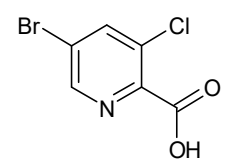

S39

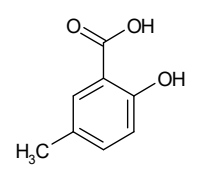

$\mathrm{S} 40$<smiles>CC(=O)Cc1cccc(C)c1</smiles>

S41

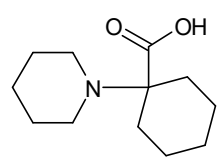

S42

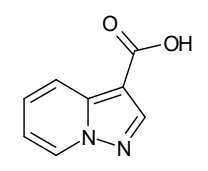


S43

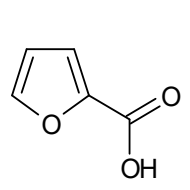

S44

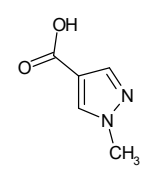

S45

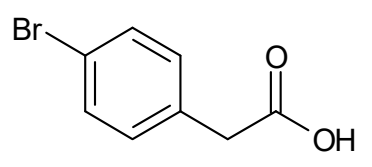

S46

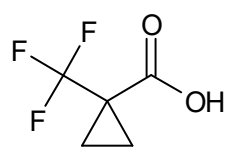

S47

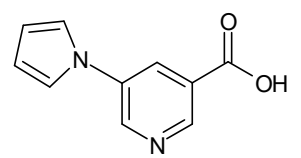

S48

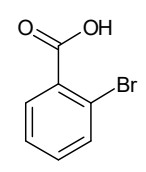

S49

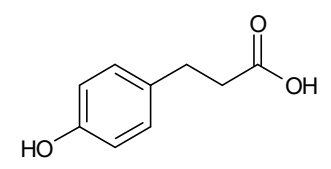

S50

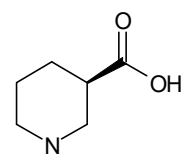

S51

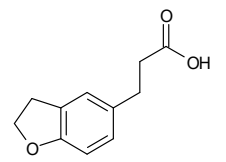

S52

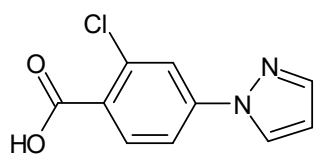

S53

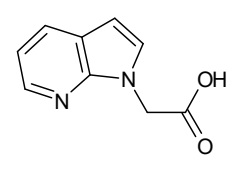


S54

S55

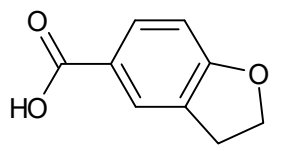

S56
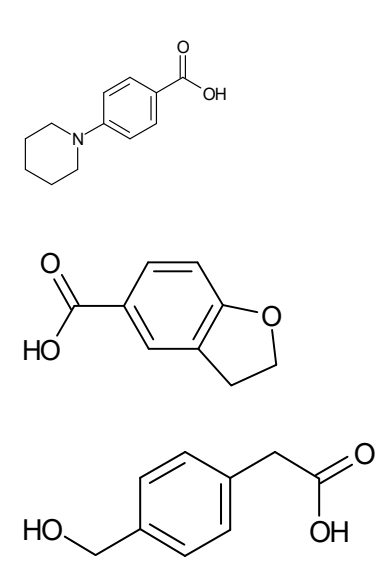

S57
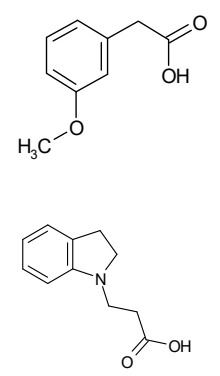

S59

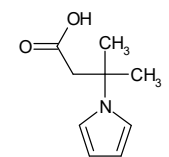

$\mathrm{S} 60$
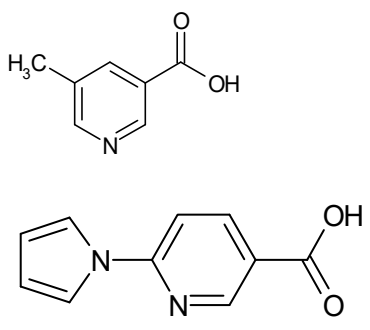

S62

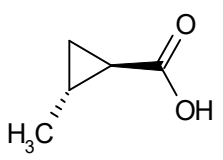

S63

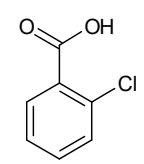

S64

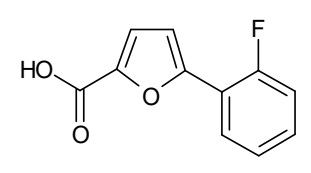




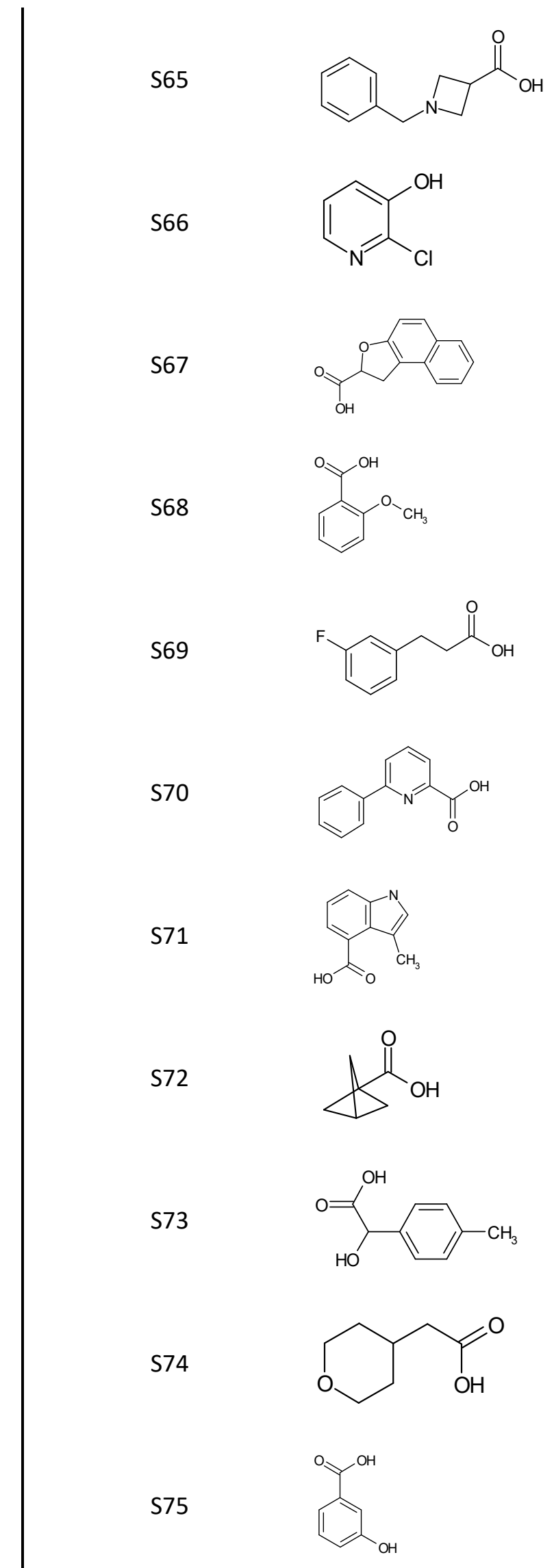


S76

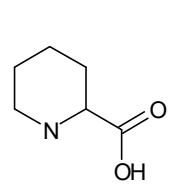

S77

S78

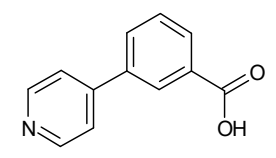

S79

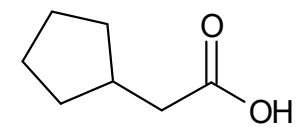

$\mathrm{S} 80$

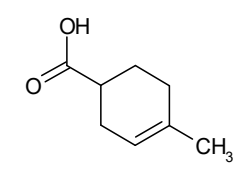

S81

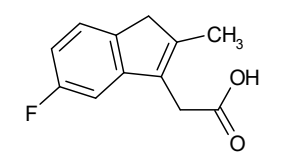

S82
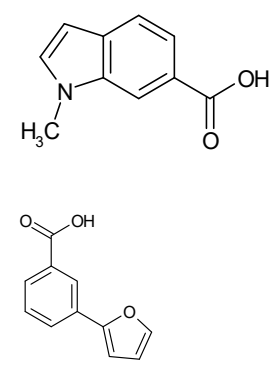

S83

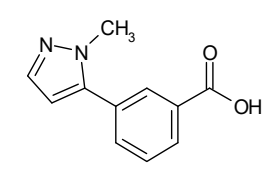

S84

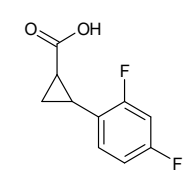

S85

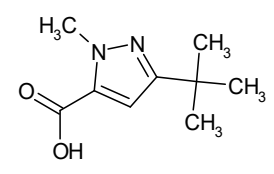

S86

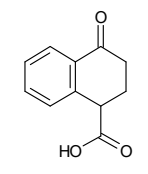


S87

S88

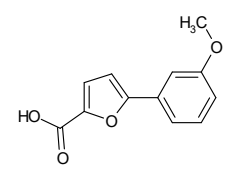

S89

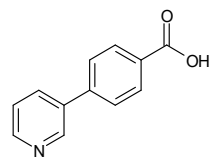

S90

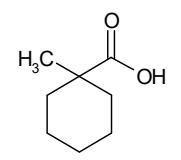

S91

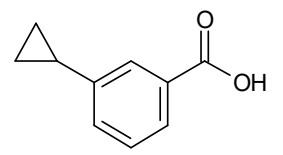

S92

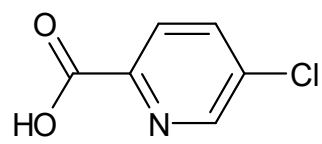

S93

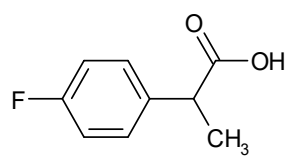

S94

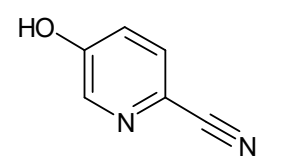

S95
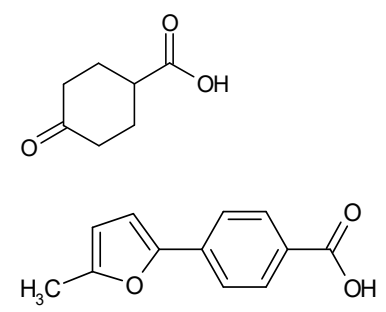

S96

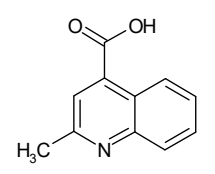

S97 
S98

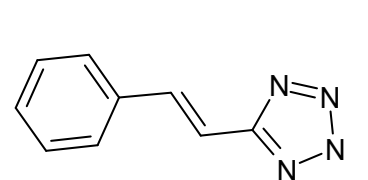

S99

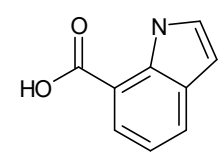

S100

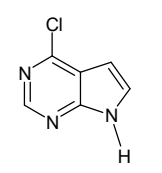

S101

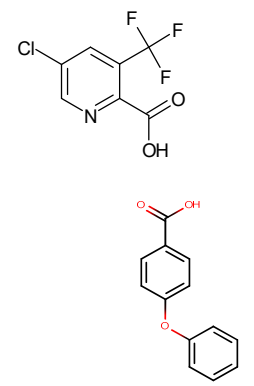

S102

S103
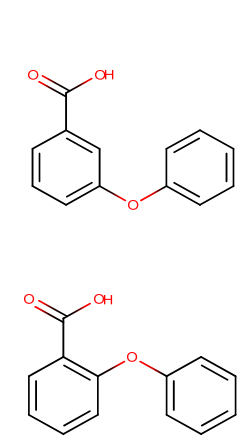

S104 


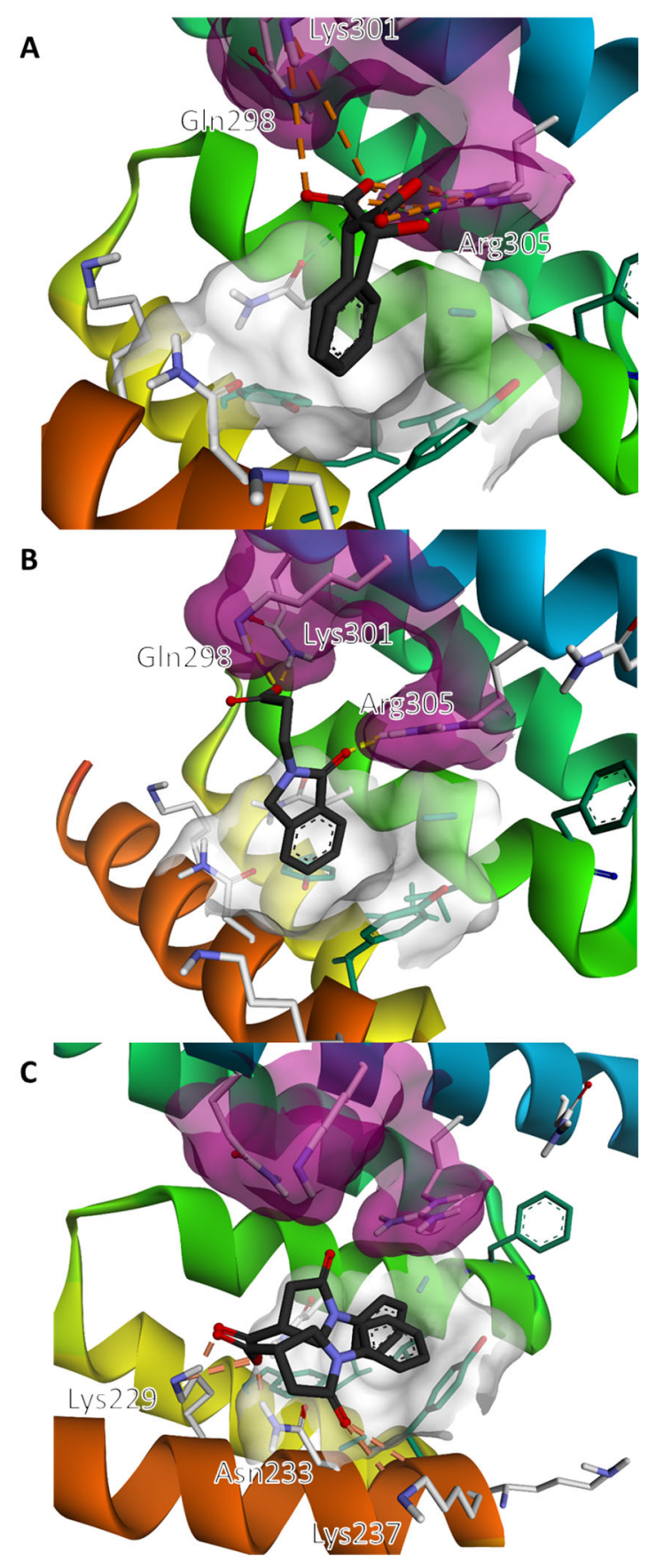

Figure S1 A - C. Docked binding pose of F23 ( $R$ and $S)$, F26 and F27 ( $R$ and $S)$ respectively. 